\title{
An electrical probe for mechanical vibrations in suspended carbon nanotubes
}

\author{
N. Traverso Ziani ${ }^{1}$, G. Piovano ${ }^{1,2}$, F. Cavaliere ${ }^{1,2}$ and M. Sassetti ${ }^{1,2}$ \\ 1 Dipartimento di Fisica, Università di Genova, \\ Via Dodecaneso 33, 16146, Genova, Italy. \\ and \\ 2 CNR-SPIN, Via Dodecaneso 33, 16146, Genova, Italy.
}

(Dated: March 8, 2021)

\begin{abstract}
The transport properties of a suspended carbon nanotube probed by means of a STM tip are investigated. A microscopic theory of the coupling between electrons and mechanical vibrations is developed. It predicts a position-dependent coupling constant, sizeable only in the region where the vibron is located. This fact has profound consequences on the transport properties, which allow to extract information on the location and size of the vibrating portions of the nanotube.

PACS numbers: $85.85 .+\mathrm{j}, 73.63 . \mathrm{Kv}$
\end{abstract}

\section{INTRODUCTION}

Carbon nanotubes (CNTs) 1 are extremely versatile systems with metallic or semiconducting behavior depending on their wrapping orientation..$[3]$ Deposing them on an insulating substrate and tunnel-coupling it to biased electrodes it is possible to create a single-electron transistor, in which the nanotube behaves as a quantum dot. ${ }^{4}[6]$ Alternately one can embed a quantum dot into the nanotube via geometrical defects or external gates, thus building a nanotube dot tunnel-coupled to contacts. ${ }^{7}$

Recent improvements in manipulation techniques have allowed to suspend nanotubes between two contacts. In this case nanotubes behave as mechanical resonators,,$\underline{8}$ with possible applications ranging from ultra-sensitive mass sensing to displacement sensors 9 Among the different mechanical vibrations 10 the radial breathing mode is the highest in energy $\sqrt{11}[13$ followed by the twist and the stretching ones. The latter have received a lot of experimental attention 14 17 also in view of the peculiar features induced on transport, such as negative differential conductance ${ }^{[15]}$ or Franck-Condon blockade ${ }^{[17}$ Bending modes have usually energies lower than the experimental temperature requiring external AC drivings. $\frac{18 \mid 19}{11}$ Transport experiments have been employed to analyze the structure of nanotubes exploiting a scanning tunneling microscope (STM) tip.20 Effects such as spincharge separation were observed studying the differential conductance as a function of the tip position. 21 Superconducting probes have been used to extract the nonequilibrium electron energy distribution function ${ }^{22}$ Also, the effects of chemical or magnetic impurities adsorbed along the nanotube were considered $[23$

Scanning tunnel microscopy experiments have also been performed on suspended nanotubes. In particular, it has been shown how electrons injected from a tunnel microscope tip can excite, detect and control a specific vibrational mode 11 13

Owing to their small waist (of the order of some $\mathrm{nm}$ ) nanotubes behave as a one-dimensional interacting electronic system. 213 Typically, correlated quantum systems are studied by means of numerical techniques ${ }^{29}$ However, due to their inherently one-dimensional nature, carbon nanotubes are described in terms of a Luttinger model $\stackrel{33134}{1 n}$ this context, transport from a tunneling tip to a static nanotube has been recently considered! $[28] 35, \frac{39}{39}$ The coupling between the electrons and vibrational modes has been extensively studied in literature 40,47 In most cases the simple Anderson-Holstein model has been employed,,$\frac{48 \mid 49}{}$ in which the vibron couples only to the total charge neglecting the spatial modulation of the charge density. The Anderson-Holstein interaction yields position-independent Franck-Condon factors $50 \mid 51$ with visible effects in transport properties. 52

Recently, a microscopic theory involving the coupling with spatial fluctuations of the nanotube electronic density has also been developed ${ }^{\sqrt{58}}$ in order to explain anomalous transport behaviors. $\frac{58}{5}$

In this paper we investigate the possibility of creating an electrical probe for the stretching vibrational modes of a suspended carbon nanotube, by means of a scanning tunnel microscope tip. Building on the theory outlined in Ref. 58, we describe the coupling between vibrons and total charge as well as the spatial charge density modulations. This coupling gives rise to a position-dependent, electron-vibron coupling which strongly affects the transport properties. Position-dependent tunneling rates and conductance arise. This allows to obtain precise informations about the vibrational mode of the nanotube. Effects are visible in metallic nanotubes and are more pronounced in semiconducting ones.

The paper is structured as follows. In Sec. IIA a Luttinger liquid model for a carbon nanotube with open boundary conditions is introduced. In Secs. IIB II C the lattice vibrations, the electron-vibron coupling and its diagonalization are discussed. Section IID is devoted to the transport properties. Our results are illustrated and commented in Sec. III] Conclusions are drawn in Sec. IV] 


\section{MODEL AND METHODS}

\section{A. Modeling a carbon nanotube quantum dot}

The electronic properties of the CNT are characterized by the wrapping vector $\mathbf{w}_{n, m}=n \mathbf{a}_{+1}+m \mathbf{a}_{-1}$, where $\mathbf{a}_{ \pm 1}$ represent the basis vectors of the graphene lattice. ${ }^{[2}$ Due to the wrapped nature of the system, the energy spectrum is composed of subbands corresponding to transverse excitations along the waist of the tubule. In a typical experiment only the lowest-lying subband is occupied, ${ }^{2[3}$ giving a one dimensional character to the CNT. In this regime both $n$-doped semiconducting CNTs (away from the band gap) and metallic CNTs can be described in the low energy sector as Luttinger liquids with four branches, $\stackrel{59}{63}$ labeled by $\alpha= \pm 1$, stemming from the two Dirac valleys of the graphene, and by $s= \pm 1$, denoting the $z$ component of the electron spin (units $\hbar / 2$ ).

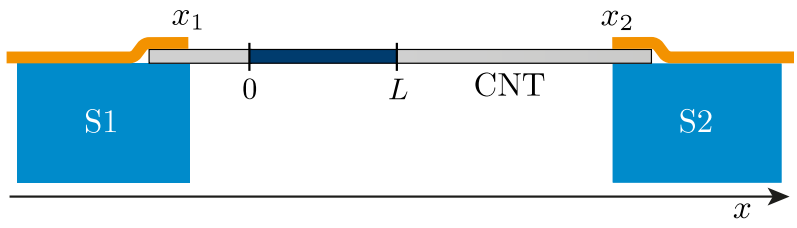

FIG. 1: (Color online) Schematic setup of a CNT, suspended between the two substrates $\mathrm{S} 1$ and $\mathrm{S} 2$ at positions $x_{1}$ and $x_{2}$. A quantum dot with ends at $x=0$ and $x=L$ is embedded in the CNT.

The system under investigation is schematically depicted in Fig. 1, it consists of a CNT, suspended between two substrates at $x_{1}$ and $x_{2}$ and free to vibrate. Embedded in the CNT there is a quantum dot of length $L$ with ends at $x=0$ and $x=L$. We assume $x_{1} \leq 0$ and $x_{2} \geq L$ in order to mimic a quantum dot inside the CNT, due to geometrical defect or external gates, or to treat the CNT itself as the quantum dot. In the following, we focus on the description of the quantum dot with open boundary conditions.

The bosonized hamiltonian is $\hat{H}=\sum_{j} \hat{H}_{j}$ with

$$
\hat{H}_{j}=\frac{1}{2} E_{j} \hat{N}_{j}^{2}+\sum_{q} \omega_{j}(q) \hat{b}_{j}^{\dagger}(q) \hat{b}_{j}(q), \quad(\hbar=1)
$$

where $j \in\left\{\rho_{+}, \rho_{-}, \sigma_{+}, \sigma_{-}\right\}$are the four linear combinations of states in the $\alpha, s$ branches that diagonalize the Coulomb interaction. Here, $\hat{N}_{j}$ represent the zero modes counting the excess electrons in the $j$ sector

$$
\begin{gathered}
\hat{N}_{\rho_{+}}=\sum_{\alpha, s} \hat{N}_{\alpha, s} \quad ; \quad \hat{N}_{\rho_{-}}=\sum_{\alpha, s} \alpha \hat{N}_{\alpha, s} \\
\hat{N}_{\sigma_{+}}=\sum_{\alpha, s} s \hat{N}_{\alpha, s} \quad ; \quad \hat{N}_{\sigma_{-}}=\sum_{\alpha, s} \alpha s \hat{N}_{\alpha, s} .
\end{gathered}
$$

The bosonic operators $\hat{b}_{j}(q)$ trigger collective excitations of the electron system with momentum $q=\pi n / L$ with

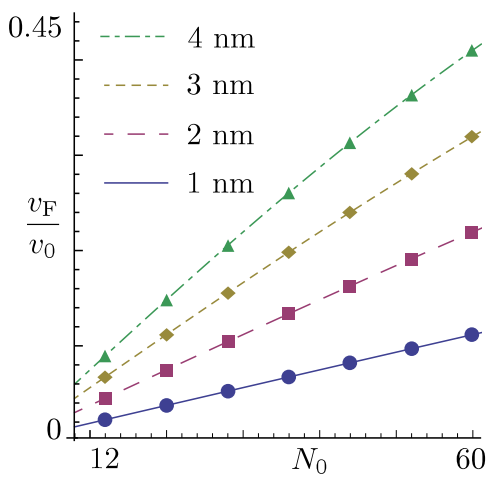

FIG. 2: (Color online) Fermi velocity $v_{\mathrm{F}}$ of a semiconducting CNT of length $L=400$ with $N_{0}$ electrons and different CNT waist lengths $\left|\mathbf{w}_{n, m}\right|$ : blue (solid) $1 \mathrm{~nm}$ - such as for $(3,2)$, purple (dashed) $2 \mathrm{~nm}$ - such as for $(5,4)$, yellow (dotted) 3 $\mathrm{nm}$ - such as for $(8,6)$, green (dash-dotted) $4 \mathrm{~nm}$ - such as for $(11,7)$.

$n \in \mathbb{N}^{*}$. They are connected to the $\alpha, s$ modes by a Bogoljubov transformation. ${ }^{59 \mid 60}$ Note that the mode $j=$ $\rho_{+}$represents the total charge of the system.

The collective modes propagates with velocities $v_{j}=$ $v_{\mathrm{F}} / g_{j}$ with $g_{\rho_{+}}=g$ and $g_{j}=1 \forall j \neq \rho_{+}$. Here $g$ parameterizes the strength of electron scattering, with $g<1$ for repulsive interactions. Note that only the velocity of the total charge mode is renormalized. The corresponding energies are $\omega_{j}(q)=v_{j} q$ and $E_{j}=\pi v_{j} / 4 g_{j} L$.

The value of the Fermi velocity $v_{\mathrm{F}}$ depends on the properties of the CNT. For a metallic CNT $(n-m \mid / 3 \in \mathbb{N})$ the dispersion relation is linear with $v_{\mathrm{F}}=v_{0}=8 \cdot 10^{5} \mathrm{~m} / \mathrm{s}$ around the degenerate point $\bar{k}$. In the following, we will assume an effective $n$-doping with a shift of $E_{\mathrm{F}}$ towards higher energy values and new Fermi points $k_{\mathrm{F}}^{( \pm)}=\bar{k} \pm q_{\mathrm{F}}$ with $q_{\mathrm{F}}=E_{\mathrm{F}} / v_{\mathrm{F}}$.

In a semiconducting CNT the conduction and valence bands are separated at momentum $\bar{k}^{\prime}$ by the direct gap 2

$$
\Delta=\frac{4 \pi v_{0}}{3\left|\mathbf{w}_{n, m}\right|},
$$

with $\left|\mathbf{w}_{n, m}\right|=a \sqrt{n^{2}+n m+m^{2}}$ the waist length of the CNT. We will consider $n$-doped semiconducting nanotubes with $E_{\mathrm{F}}>\Delta / 2$, having chosen the energy reference to lie in the middle of the band gap. Doping gives rise to two new Fermi points $k_{\mathrm{F}}^{( \pm)}=\bar{k}^{\prime} \pm q_{\mathrm{F}}$ where $q_{\mathrm{F}}=2 m^{*} \sqrt{E_{\mathrm{F}}-\Delta}$ with $m^{*}=\Delta / 2 v_{0}^{2}$ the effective mass. ${ }^{2}$ Around the new Fermi points, the Fermi velocity is

$$
v_{\mathrm{F}}=\frac{3\left|\mathbf{w}_{n, m}\right| N_{0}}{8 L},
$$

with $N_{0}$ the total number of excess electrons in the quantum dot. The dependence of $v_{\mathrm{F}}$ as a function of $N_{0}$ is shown in Fig. 2. One observes a lower velocity with respect to the metallic case. 
The electron field operator $\hat{\Psi}_{s}(\mathbf{r}) \equiv \hat{\Psi}_{s}(x, y)$ has to satisfy open-boundaries conditions $\hat{\Psi}_{s}(0, y)=\hat{\Psi}_{s}(L, y)=0$. It can be written in the bosonized form

$$
\hat{\Psi}_{s}(\mathbf{r})=\sum_{r= \pm 1} \sum_{\alpha= \pm 1} f_{r, \alpha}(\mathbf{r}) e^{i r q_{\mathrm{F}} x} \hat{\psi}_{+1, r \alpha, s}(r x)
$$

in terms of the right-movers field operators

$$
\begin{aligned}
\hat{\psi}_{+1, \alpha, s}(x)= & \frac{\hat{\eta}_{\alpha, s}}{\sqrt{2 \pi \tilde{a}}} e^{-i \theta_{\alpha, s}} e^{i \frac{\pi x}{4 L}\left(\hat{N}_{\rho_{+}}+\alpha \hat{N}_{\rho_{-}}+s \hat{N}_{\sigma_{+}}+\alpha s \hat{N}_{\sigma_{-}}\right)} . \\
& e^{\frac{i}{2}\left[\hat{\phi}_{\rho_{+}}(x)+\alpha \hat{\phi}_{\rho_{-}}(x)+s \hat{\phi}_{\sigma_{+}}(x)+\alpha s \hat{\phi}_{\sigma_{-}}(x)\right]}
\end{aligned}
$$

where

$$
\begin{aligned}
\hat{\phi}_{j}(x) & =\sum_{q} \sqrt{\frac{\pi}{q L}}\left\{\frac{1}{\sqrt{g_{j}}} \cos (q x)\left[\hat{b}_{j}(q)+\hat{b}_{j}^{\dagger}(q)\right]\right. \\
& \left.+i \sqrt{g_{j}} \sin (q x)\left[\hat{b}_{j}(q)-\hat{b}_{j}^{\dagger}(q)\right]\right\} .
\end{aligned}
$$

Here $\hat{\eta}_{\alpha, s}$ are Majorana fermions, $\left[\hat{\theta}_{\alpha, s}, \hat{N}_{\alpha, s}\right]=i$, and $\tilde{a}$ is the length cutoff. The functions $f_{r, \alpha}(\mathbf{r})$ in Eq. (4) consist of a superposition of wavefunctions for $p_{z}$ orbitals, peaked around the positions of atoms in the CNT and oscillating with a typical wave vector $K_{0} \propto a^{-1}$ where $a \approx 2.5 \cdot 10^{-10} \mathrm{~m} !^{[5960]}$ Their specific form depends on the type of nanotube under consideration and will be not discussed here.

\section{B. Lattice vibrations and electron-vibron coupling}

We consider the case of a vibrating portion of the CNT (the vibron), of size $\mathcal{L}$, located between $x_{v 0}$ and $x_{v 1}$. As confirmed in a recent experiment, 58 the vibrating part can be different from the CNT dot. Thus, we will formulate the theory in this most general case. We focus the description on the stretching mode. The $p$-th mode has energy $\omega_{0}=p \pi v_{\mathrm{s}} / \mathcal{L}$ with $v_{\mathrm{s}} \approx 2.4 \cdot 10^{4} \mathrm{~m} / \mathrm{s}$ the velocity of the stretching modes which is approximately nondispersive. In most experiments the fundamental mode with $p=1$ is observed 1517 For these reasons, we will concentrate to the case of small $p \leq 3$. The $p$-th mode is described as a harmonic oscillator

$$
\hat{H}_{\mathrm{v}}=\frac{\hat{P}_{0}^{2}}{2 M}+\frac{M \omega_{0}^{2}}{2} \hat{X}_{0}^{2},
$$

where $M=2 \pi\left|\mathbf{w}_{n, m}\right| \mathcal{L} \rho_{0}$ is the vibron mass with $\rho_{0} \approx$ $6.7 \cdot 10^{-7} \mathrm{Kg} / \mathrm{m}^{2}$ the graphene density and $\hat{X}_{0}$ is the amplitude operator of the strain field

$$
\hat{u}_{p}(\mathbf{r})=\sqrt{2} \hat{X}_{0} \sin \left[p \frac{\pi\left(x-x_{v 0}\right)}{\mathcal{L}}\right] .
$$

The latter represents a standing wave with momentum $\pm q_{0}$ with $q_{0}=p \pi / \mathcal{L}$.
The coupling between electrons and vibrations can be microscopically derived starting from the tight-binding theory of a distorted CNT lattice. ${ }^{42 / 64}$ For the typical experimental situations one has $\mathcal{L} \gtrsim 100 \mathrm{~nm}$ then a continuum elastic model is appropriate with

$$
H_{\mathrm{d}-\mathrm{v}}=c \int \mathrm{d} \mathbf{r} \hat{\rho}(\mathbf{r}) \partial_{x} \hat{u}_{p}(\mathbf{r}) \text {, }
$$

Here $e^{40} c \approx 30 \mathrm{eV}$ and $\hat{\rho}(\mathbf{r})=\sum_{s} \hat{\Psi}_{s}^{\dagger}(\mathbf{r}) \hat{\Psi}_{s}(\mathbf{r})$ is the electronic density operator, with $\hat{\Psi}_{s}(\mathbf{r})$ given by Eq. (4). It consists of two components: a long wavelength part $\rho_{\mathrm{LW}}(\mathbf{r})$ and an oscillatory contribution $\rho_{\mathrm{SW}}(\mathbf{r})$ fluctuating on a length scale $K_{0}^{-1} \propto a$. This latter component, does not make sizeable contributions since $q_{0} \ll$ $K_{0}$. Under the realistic assumption of strongly localized atomic orbitals ${ }^{2 \mid 3}$ with negligible overlapping, the electron-vibron coupling becomes

$$
H_{\mathrm{d}-\mathrm{v}}=c \int_{x_{<}}^{x_{>}} \mathrm{d} x \hat{\rho}_{\mathrm{LW}}(x) \partial_{x} \hat{u}_{p}(x) \text {, }
$$

with $x_{<}=\max \left\{0, x_{v 0}\right\}, x_{>}=\min \left\{x_{v 1}, L\right\}$ and

$$
\hat{\rho}_{\mathrm{LW}}=\frac{\hat{N}_{\rho_{+}}}{L}+\frac{1}{2 \pi}\left[\partial_{x} \hat{\phi}_{\rho_{+}}(x)+x \rightarrow-x\right],
$$

written here directly in its bosonized form.

\section{Diagonalizing the electron-vibron coupling}

The relevant terms of the electron-vibron coupling are $\hat{h}=\hat{H}_{\rho_{+}}^{(0)}+\hat{H}_{\rho_{+}}^{(\mathrm{pl})}+\hat{H}_{\mathrm{v}}+\hat{H}_{\mathrm{d}-\mathrm{v}}$ with $H_{\rho_{+}}^{(0)}=E_{\rho_{+}} \hat{N}_{\rho_{+}}^{2} / 2$ and $\hat{H}_{\rho_{+}}^{(\mathrm{pl})}=\sum_{q} \omega_{\rho_{+}}(q) \hat{b}_{\rho_{+}}(q) \hat{b}_{\rho_{+}}(q)$. Introducing $\hat{B}_{\mu}=$ $i \hat{b}_{\rho_{+}}(\pi \mu / L)$ and $\sqrt{2 \omega_{\rho_{+}}(\pi \mu / L)} \hat{X}_{\mu}=\hat{B}_{\mu}+\hat{B}_{\mu}^{\dagger}$ we have

$$
\begin{aligned}
\hat{h} & =\frac{1}{2} E_{\rho_{+}} \hat{N}_{\rho_{+}}^{2}+\frac{\hat{P}_{0}^{2}}{2 M}+\frac{M \omega_{0}^{2}}{2} \hat{X}_{0}^{2}+\sum_{\mu \geq 1}\left(\frac{\hat{P}_{\mu}^{2}}{2}+\omega_{\mu}^{2} \frac{\hat{X}_{\mu}^{2}}{2}\right) \\
& +\sqrt{M} C_{0} \hat{X}_{0} \hat{N}_{\rho_{+}}+\sqrt{M} \hat{X}_{0} \sum_{\mu \geq 1} C_{\mu} \hat{X}_{\mu},
\end{aligned}
$$

with $\left[\hat{X}_{\mu}, \hat{P}_{\nu}\right]=i \delta_{\mu, \nu}, \omega_{\mu}=\mu \omega_{1} / g, \omega_{1}=\pi v_{\mathrm{F}} / L$. In terms of these new variables the density operator is

$$
\hat{\rho}_{\mathrm{LW}}(x)=\frac{\hat{N}_{\rho_{+}}}{L}+\sqrt{\frac{2 \pi v_{\mathrm{F}}}{L^{3}}} \sum_{\mu \geq 1} \mu \cos \left(\frac{\pi \mu x}{L}\right) \hat{X}_{\mu} .
$$

The last term in Eq. (12) describes a central harmonic oscillator (vibron) linearly coupled to a infinity of harmonic oscillators (plasmon modes of the dot). Note that, for reasonable experimental parameters and considering the lowest stretching modes, one always has $\omega_{\mu}>\omega_{0}$ both for metallic and semiconducting CNTs. Additionally, the vibron is also coupled to the total average charge $\hat{N}_{\rho_{+}}$on 
the quantum dot in analogy to the Anderson-Holstein model. The coupling coefficients are

$$
C_{0}=\sqrt{2} \lambda_{\mathrm{m}} \omega_{0}^{3 / 2} J_{0} \quad ; \quad C_{\mu \geq 1}=2 \lambda_{\mathrm{m}} \omega_{0}^{3 / 2} \sqrt{\omega_{1}} J_{\mu}
$$

where $(\kappa \geq 0)$

$$
J_{\kappa}=\frac{1}{L} \int_{x_{<}}^{x_{>}} \mathrm{d} x \cos \left[\frac{\kappa \pi x}{L}\right] \cos \left[\frac{p \pi}{\mathcal{L}}\left(x-x_{v 0}\right)\right],
$$

and

$$
\lambda_{\mathrm{m}}=\frac{c}{v_{\mathrm{s}} \sqrt{\rho_{0} \pi\left|\mathbf{w}_{n, m}\right| v_{\mathrm{s}}}} .
$$

Taking as a reference ${ }^{2 / 3}$ a waist length of about $2 \mathrm{~nm}$, one has $\lambda_{\mathrm{m}} \approx 2$. Note that a recent experiment ${ }^{65}$ reports a larger $c$ which leads to a larger $\lambda_{\mathrm{m}}$. One finds

$$
J_{\kappa}=J_{\kappa}^{(0)}+J_{\kappa}^{(-)} \theta\left(-0^{+}-x_{v 0}\right)+J_{\kappa}^{(+)} \theta\left(x_{1}-L-0^{+}\right),
$$

with $\theta(x)$ the Heavyside step function and

$$
\begin{aligned}
J_{\kappa}^{(0)} & =\frac{\kappa \delta^{2}\left\{\sin \left(\pi \kappa \xi_{0}\right)-(-1)^{p} \sin \left[\pi \kappa\left(\xi_{0}+\delta\right)\right]\right\}}{\pi\left(p^{2}-\kappa^{2} \delta^{2}\right)}, \\
J_{\kappa}^{(-)} & =\frac{p \delta \sin \left(\pi p \xi_{0} / \delta\right)-\kappa \delta^{2} \sin \left(\pi \kappa \xi_{0}\right)}{\pi\left(p^{2}-\kappa^{2} \delta^{2}\right)}, \\
J_{\kappa}^{(+)} & =\frac{(-1)^{p} \kappa \delta^{2} \sin \left[\pi \kappa\left(\xi_{0}+\delta\right)\right]}{\pi\left(p^{2}-\kappa^{2} \delta^{2}\right)} \\
& -\frac{p \delta(-1)^{\kappa} \sin \left[\pi p \delta\left(1-\xi_{0}\right) / \delta\right]}{\pi\left(p^{2}-\kappa^{2} \delta^{2}\right)},
\end{aligned}
$$

where $\delta=\mathcal{L} / L$ and $\xi_{0}=x_{v 0} / L$.

Let us now comment the general features of the coupling depending on the relative size and position of dot and vibron. When the vibron is much larger than the $\operatorname{dot}(\delta \gg 1)$ and the latter is embedded into it one has $J_{\kappa} \approx \delta_{\kappa, 0}$, namely in the large vibron limit the coupling to the charge density fluctuations vanishes and only the conventional Anderson-Holstein coupling survives. In this limit, the electron-vibron coupling reduces to the standard form $\omega_{0} \lambda_{\mathrm{m}} \ell_{0}^{-1} \hat{X}_{0} \hat{N}_{\rho_{+}}$with $\ell_{0}^{-1}=\sqrt{M \omega_{0}}$, and the coupling constant is $\lambda_{\mathrm{m}}$.

The most interesting case occurs when the vibron is smaller than the dot and embedded into it. In this regime, one finds $J_{0} \equiv 0$, while $J_{\kappa \geq 1} \neq 0$. This fact signals a radical departure from the Anderson-Holstein model: the coupling between electrons and vibrons occurs only via the spatial fluctuations of the electron density.

We now turn to the diagonalization of the electron-vibron coupling. The terms linear in $\hat{X}_{\mu}$ in Eq. 12 can be exactly diagonalized, $\stackrel{67}{,}$ leading to

$$
\begin{aligned}
\hat{h} & =\frac{1}{2} E_{\rho_{+}} \hat{N}_{\rho_{+}}^{2}+\sum_{\mu \geq 0}\left(\frac{\hat{\bar{P}}_{\mu}^{2}}{2}+\Omega_{\mu}^{2} \frac{\hat{\bar{X}}_{\mu}^{2}}{2}\right) \\
& +\sqrt{M} C_{0}\left(\sum_{\nu \geq 0} k_{0 \nu} \hat{\bar{X}}_{\nu}\right) \hat{N}_{\rho_{+}},
\end{aligned}
$$

with

$$
\left(\hat{X}_{\mu}, \hat{P}_{\mu}\right)=\sum_{\nu \geq 0} k_{\mu \nu}\left(\hat{\bar{X}}_{\nu}, \hat{\bar{P}}_{\nu}\right)
$$

In order to diagonalize the term $\propto \hat{N}_{\rho_{+}}$a Lang-Firsov canonical transformation is used

$$
\hat{\mathcal{U}}=e^{-i \hat{N}_{\rho_{+}} \sum_{\nu \geq 0} \eta_{\nu} \hat{\bar{P}}_{\nu}}
$$

with $\eta_{\nu}=\sqrt{M} C_{0} k_{0 \nu} /\left(\Omega_{\nu}^{2} \sqrt{M}\right)$. This leads to a shift

$$
\hat{\bar{X}}_{\nu} \rightarrow \hat{\bar{X}}_{\nu}-\eta_{\nu} \hat{N}_{\rho_{+}}
$$

which finally casts the hamiltonian into the diagonal form

$$
\hat{h}=\frac{1}{2}\left(E_{\rho_{+}}-\Delta E_{\rho_{+}}\right) \hat{N}_{\rho_{+}}^{2}+\sum_{\mu \geq 0}\left(\frac{\hat{\bar{P}}_{\mu}^{2}}{2}+\Omega_{\mu}^{2} \frac{\hat{\bar{X}}_{\mu}^{2}}{2}\right),
$$

with $\Delta E_{\rho_{+}}=C_{0}^{2} \sum_{\nu \geq 0}\left(k_{0 \nu}^{2} / \Omega_{\nu}^{2}\right)$.

The energies $\Omega_{\mu}$ of the new eigenmodes are the roots of the secular equation

$$
z^{2}=\omega_{0}^{2}+\sum_{\nu \geq 1} \frac{C_{\nu}^{2}}{z^{2}-\nu^{2} \omega_{1}^{2}}
$$

with

$$
\begin{aligned}
& k_{\mu \nu}=\frac{C_{\mu}}{\Omega_{\nu}^{2}-\mu^{2} \omega_{1}^{2}} k_{0 \nu} \quad(\text { with } \mu \geq 1), \\
& k_{0 \nu}=\left[1+\sum_{\mu \geq 1} \frac{C_{\mu}^{2}}{\left(\Omega_{\nu}^{2}-\mu^{2} \omega_{1}^{2}\right)^{2}}\right]^{-1 / 2} .
\end{aligned}
$$

As can be clearly seen, the modes with $\mu \geq 1$ have
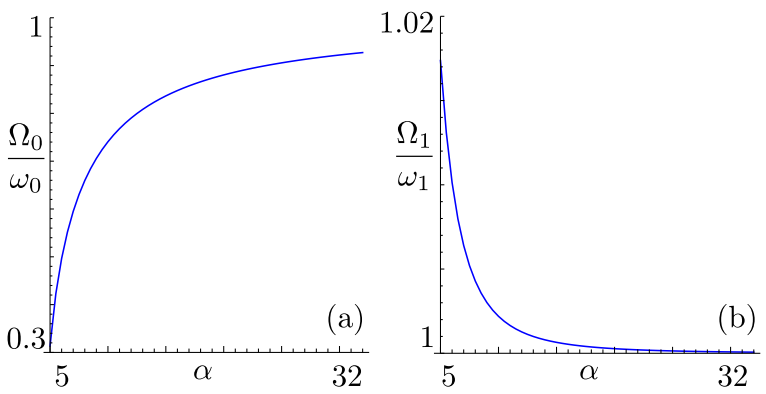

FIG. 3: Energy of the lowest eigenmode. (a) Plot of $\Omega_{0} / \omega_{0}$ as a function of $\alpha$; (b) Plot of $\Omega_{1} / \omega_{1}$ as a function of $\alpha$. In all figures, $\delta=1, x_{v 0}=0, p=1, g=1$ and $\lambda_{\mathrm{m}}=2$.

energies $\Omega_{\mu} \gtrsim \mu \omega_{1}$ and represent blue-shifted dressed plasmons. The lowest-lying solution, on the other hand, has $\Omega_{0}<\omega_{0}$ and represents a dressed vibronic mode red-shifted by the electron-vibron interaction. By inspecting Eq. 23) one always obtains a real solution 
for $\Omega_{\mu}$ with $\mu \geq 1$. On the other hand, the existence of a real solution for $\Omega_{0}$ requires $\omega_{0}^{2} \omega_{1}^{2}>\sum_{\mu>1} C_{\mu}^{2} / \mu^{2}$. When this condition is not fulfilled, the Wentzel-Bardeen

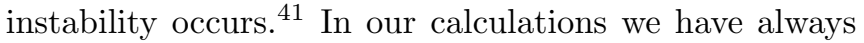
checked that for realistic parameters the system does not exhibit this instability. The energy of the dressed vibronic mode is very sensitive to the ratio $\alpha=v_{\mathrm{F}} / v_{\mathrm{s}}$ between the Fermi and the sound velocity. While for $\alpha=32$, corresponding to the case of a metallic CNT, one has $\Omega_{0} \approx \omega_{0}$, for lower values of $\alpha$, typical of a semiconducting CNT, a suppression of $\Omega_{0}$ occurs, see Fig. 3(a). Note that the dressed plasmons are almost insensitive to the ratio $\alpha$ (cf. Fig. $3(\mathrm{~b})$ ).

The transformations in Eq. (19) and 20 affects the electronic field operator of Eq. (5). Up to an irrelevant phase constant, the field $\hat{\phi}_{\rho_{+}}(x)$ is

$$
\hat{\phi}_{\rho_{+}}(x)=\sum_{\mu \geq 0} \alpha_{\mu}(x) \hat{\bar{X}}_{\mu}+\beta_{\mu}(x) \hat{\bar{P}}_{\mu},
$$

where

$$
\begin{aligned}
& \alpha_{\mu}(x)=\sqrt{2 \omega_{1}} \sum_{\nu \geq 1} k_{\nu \mu} \sin \left(\frac{\pi \nu x}{L}\right) \\
& \beta_{\mu}(x)=\sqrt{M} \eta_{\mu}+\sqrt{\frac{2}{\omega_{1}}} \sum_{\nu \geq 1} \frac{k_{\nu \mu}}{\nu} \cos \left(\frac{\pi \nu x}{L}\right) .
\end{aligned}
$$

\section{Modeling transport}

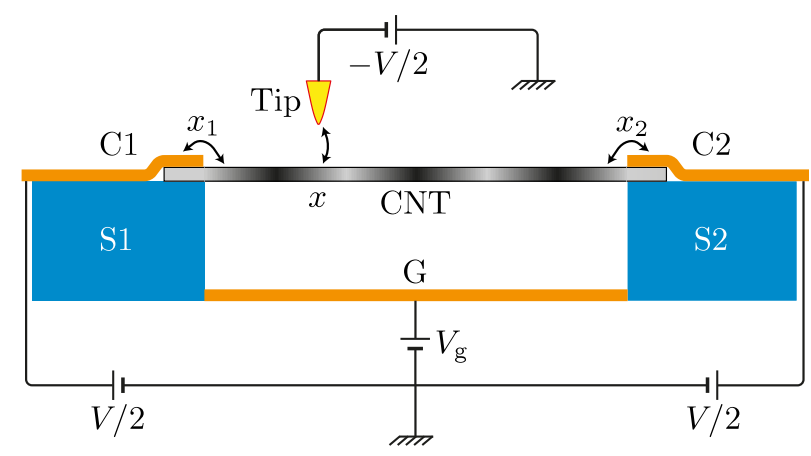

FIG. 4: Schematic setup of a STM transport experiment performed on a suspended CNT. The quantum dot is the nanotube itself, biased with respect to the tip and two lateral contacts. Electrons can flow through the system via the STM tip at position $(x)$ and at the contacts $\left(x_{1,2}\right)$. A back gate allows to tune the effective charge on the dot.

Our task is to model an STM tunneling tip on a CNT. ${ }^{11} 13$ The setup is sketched in Fig. 4 and is composed of a STM tip and two lateral contacts tunnelcoupled to a suspended CNT. Tip and contacts are biased in such a way that, for $V>0$, electrons flow from the tip to the contacts through the CNT. Additionally, a back gate is capacitively coupled to tune the effective charge on the CNT-dot. From now on, we will focus on the most interesting regime, namely that of a quantum dot along all the CNT with a vibron imbedded into it. The hamiltonian for the vibrating $\mathrm{CNT}$ is then

$H_{\mathrm{CNT}}=\frac{1}{2} E_{\rho_{+}}\left(\hat{N}_{\rho_{+}}-N_{\mathrm{g}}\right)^{2}+\sum_{\mu \geq 0} \frac{\hat{\bar{P}}_{\mu}^{2}}{2}+\frac{\Omega_{\mu}^{2}}{2} \hat{\bar{X}}_{\mu}^{2}+\sum_{j \neq \rho_{+}} \hat{H}_{j}$,

where $N_{\mathrm{g}}$ represents the charge induced by the back gate voltage $V_{\mathrm{g}}$. The dot is laterally coupled to the two Fermi contacts, via the tunneling hamiltonian 58

$$
\hat{H}_{\mathrm{T}, \mathrm{L}}=t_{0} \sum_{j=1,2} \sum_{\alpha, s, q} \hat{\psi}_{+1, \alpha, s}\left(x_{j}\right) \hat{c}_{j, s}(q)+\text { h.c. },
$$

where $t_{0}$ is the tunneling amplitude, $\hat{c}_{j, s}(q)$ are the operators for an electron with momentum $q$ and spin $s$ in the non-interacting lead $j$ and $x_{1}=0, x_{2}=L$ are the position of the tunneling contacts.

The STM tip is modeled as a semi-infinite Fermi contact, placed above the CNT at a position $x$ along it. The tunnel coupling is expressed in terms of the Fermi field operator for the forward modes of the tip $28 \mid 35 \hat{\psi}_{s, \mathrm{~F}}(z)(z$ is the coordinate along the tip with $z=0$ at the vertex)

$\hat{H}_{\mathrm{T}, \mathrm{T}}=\left[\int_{0}^{L} \mathrm{~d} y \tau_{0}(y-x) \sum_{\alpha, s} \hat{\psi}_{+1, \alpha, s}^{\dagger}(y)\right] \hat{\psi}_{s, \mathrm{~F}}\left(0^{+}\right)+$h.c..

The function $\tau_{0}(x)=\tau_{0} \varphi(x)$ describes the geometry of the tip, with $0 \leq \varphi(x) \leq 1$ peaked around $x=0$, where $\varphi(0)=1$. In the following we will assume a typical tip, with an effective width of a few atomic cells of the CNT. A voltage $-V / 2$ is applied to the STM tip, while the lateral contacts are kept at the same voltage $V / 2$. Voltage drops are assumed to occur symmetrically on the CNT. More general potential distributions do not affect the results at a qualitative level.

We will consider the sequential tunneling regime, treating the tunnel couplings to the lowest perturbative order. Since we are interested into the low-energy transport regime $\left(e|V| k_{\mathrm{B}} T \approx \Omega_{0}\right)$ we disregard the dynamics of the modes $\mu \geq 1$. Furthermore, we will consider the relevant situation of a damped vibronic mode, with a thermal equilibrium distribution at temperature $T$.

The eigenstates of the suspended CNT can be expressed by $\left|\left\{N_{\alpha, s}\right\}\right\rangle$ specifying the distribution of excess electrons in the channel $\alpha$ with spin $s$. We will consider the resonance between the state of a closed shell with $N_{0}=4 \kappa,(\kappa$ integer $)$ electrons and zero excess charges, denoted as $|0\rangle=|0,0,0,0\rangle$, and $N_{0}+1$ electrons in the state $\alpha, s$. Note that no qualitative difference in our results would occur, for a different value of $N_{0}$. There are four states with $N_{0}+1$, electrons, denoted by $|\alpha, s\rangle$, each with one extra electron in the state $\alpha, s$. They are all degenerate, with energy $E_{\rho_{+}}\left(1-N_{\mathrm{g}}\right)^{2} / 2+3 \omega_{1} / 8$. 
We set up a master equation for the reduced density matrix, obtained tracing out the leads and vibron degrees of freedom - thus neglecting coherences among vibrational states. $66|68| 69]$ Upon the assumption of a STM tip width of some unit cells, coherence effects between states $|\alpha, s\rangle$ and $\left|\alpha^{\prime}, s\right\rangle\left(\alpha \neq \alpha^{\prime}\right)$ are vanishing. Coherence between different spin states is also absent in view of the absence of spin correlations in the contacts. Therefore, the master equation reduces to a standard rate equation for the occupation probability of the quantum $\operatorname{dot} P_{N_{0}}(t)=P_{|0\rangle}(t) ; \quad P_{N_{0}+1}(t)=\sum_{\alpha, s} P_{|\alpha, s\rangle}(t)$. The steady-state current is then written, to lowest order, in terms of tunneling rates

$$
I(x)=e \frac{\Gamma_{\text {out }}^{(\mathrm{C})} \Gamma_{\text {in }}^{(\mathrm{T})}(x)-\Gamma_{\text {in }}^{(\mathrm{C})} \Gamma_{\text {out }}^{(\mathrm{T})}(x)}{\Gamma_{\mathrm{in}}(x)+\Gamma_{\mathrm{out}}(x)},
$$

with

$$
\Gamma_{\text {in } / \text { out }}(x)=\Gamma_{\text {in } / \text { out }}^{(\mathrm{T})}(x)+\Gamma_{\text {in } / \text { out }}^{(\mathrm{C})}
$$

and

$$
\begin{aligned}
& \Gamma_{\text {in }}^{(\mathrm{T})}(x)=\sum_{\alpha, s} \Gamma_{|0\rangle \rightarrow|\alpha, s\rangle}^{(\mathrm{T})}(x) ; \quad \Gamma_{\text {out }}^{(\mathrm{T})}(x)=\Gamma_{|\alpha, s\rangle \rightarrow|0\rangle}^{(\mathrm{T})}(x), \\
& \Gamma_{\text {in }}^{(\mathrm{C})}=\sum_{j} \sum_{\alpha, s} \Gamma_{|0\rangle \rightarrow|\alpha, s\rangle}^{(j)} ; \quad \Gamma_{\text {out }}^{(\mathrm{C})}=\sum_{j} \Gamma_{|\alpha, s\rangle \rightarrow|0\rangle}^{(j)},
\end{aligned}
$$

the rate associated to the tip $(\mathrm{T})$ and to the contacts $(\mathrm{C})$. We quote here explicitly the expressions for the tunnel-in processes, the tunnel-out rates are similar.

The lateral contact and tip rates are respectively

$$
\begin{aligned}
\Gamma_{|0\rangle \rightarrow|\alpha, s\rangle}^{(j)} & =\Gamma_{0} \sum_{l \geq 0} B_{l}\left(x_{j}\right) f\left(\Delta E+l \Omega_{0}+e V / 2\right),(32) \\
\Gamma_{|0\rangle \rightarrow|\alpha, s\rangle}^{(\mathrm{T})}(x) & =\Gamma_{0}^{(\mathrm{T})} \sum_{l \geq 0} B_{l}(x) f\left(\Delta E+l \Omega_{0}-e V / 2\right)
\end{aligned}
$$

Here, $\Gamma_{0}=2 \pi \nu_{0}\left|t_{0}\right|^{2}, \Gamma_{0}^{(\mathrm{T})}=2 \pi \nu_{0}\left|\tau_{0}\right|^{2}, \nu_{0}$ is the leads density of state and $f(E)$ the Fermi function. The rates are then a superposition of Fermi functions at energies

$$
\Delta E=E_{\rho_{+}}\left(\frac{1}{2}-N_{\mathrm{g}}\right)+\frac{3}{8} \omega_{1},
$$

shifted by the energy $l \Omega_{0}$, representing the contribution of a transport channel exciting $l$ vibron quanta. The weights $B_{l}(x)$ considered in the regime $k_{\mathrm{B}} T \ll \Omega_{0}$ are $\mathrm{e}^{52}$

$$
B_{l}(x)=\frac{\lambda^{2 l}(x)}{l !} e^{-\lambda^{2}(x)},
$$

where

$$
\lambda^{2}(x)=\frac{1}{2 \Omega_{0}} \alpha_{0}^{2}(x)+\frac{\Omega_{0}}{2} \beta_{0}^{2}(x)
$$

represents the local electron-vibron couplingstrength, 58 see Eqns. (27|28). This is in sharp contrast to the case of the Anderson-Holstein model, $\sqrt[52 \sqrt[54]{56}]{5}$ appropriate for large vibrons. In that case, as discussed above, coupling to the density fluctuations would vanish leading to $\alpha_{0}(x)=\beta_{0}(x)=0$ and to a coupling simply given by $\lambda_{\max }$ which is clearly independent of the tunneling position.

\section{RESULTS}

\section{A. Local electron-vibron coupling}

Let us analyze in details the space dependence of the electron-vibron coupling $\lambda(x)$ which determines the current behavior. The coupling $\lambda(x)$ depends both on geometrical parameters $x_{v 0}$ (the vibron origin), $\delta=\mathcal{L} / L$ (vibron length) and on physical ones $\alpha=v_{\mathrm{F}} / v_{\mathrm{s}}, \lambda_{\mathrm{m}}$ defined in Eq. (16) and the electronic interaction parameter $g$.

We remind that the parameter $\alpha$ is affected by the metallic or semiconducting nature of the CNT. In a metallic CNT one finds $\alpha=32$. On the other hand, for a semiconducting CNT lower values of $\alpha$ are possible (see later). Figure 5 shows $\lambda(x)$ for different vibron configurations
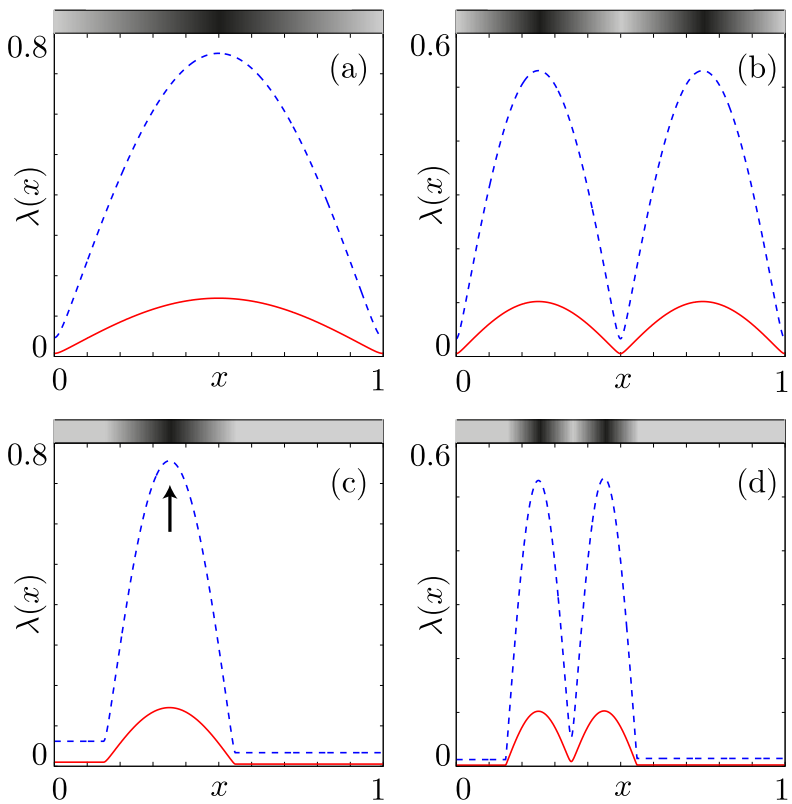

FIG. 5: (Color online) Local electron-vibron coupling $\lambda(x)$ as a function of the tip position $x$ for: (a) $\delta=1, x_{v 0}=0, p=1$; (b) as above but $p=2$; (c) $\delta=0.4, x_{v 0}=0.15 L, p=1$; (d) as above but $p=2$. In all panels red (solid) lines denote a metallic CNT with $\alpha=32$, blue (dashed) lines denote a semiconducting CNT with $\alpha=5$. Other parameters: $\lambda_{\mathrm{m}}=2$ and $g=1$. The shaded plots on top of the panels depict the amplitude of the strain field $\hat{u}_{p}(x)$. The arrow in Panel (c) denotes the tip position for the conductance shown in Fig. 8.

and CNT types. It can be seen that the electron-vibron 
coupling strength for a metallic CNT (solid red lines) is smaller than that for a semiconducting CNT. Indeed, for a semiconducting CNT, the velocities of the electronic and vibronic subsystems are closer, which implies a more favorable interplay between them. In the rest of the paper, we will choose $\alpha=5$ to model a semiconducting CNT and $\alpha=32$ for the metallic one.

The amplitude of the electron-vibron coupling is maximal in the region where the strain field is maximum. Indeed, $\lambda(x)$ closely follows the amplitude of $\hat{u}_{p}(x)$, which is sketched on top of the panels of Fig. 5 . For $\delta<1$, this implies a particularly sizeable $\lambda(x)$ only in the region where the vibron sits, see Figs. 5(c,d). Coupling to higher vibronic modes produces more oscillations, as can be seen in Figs. 5(b,d). It also makes the electron-vibron coupling strength weaker. Figure 6 shows the comparison

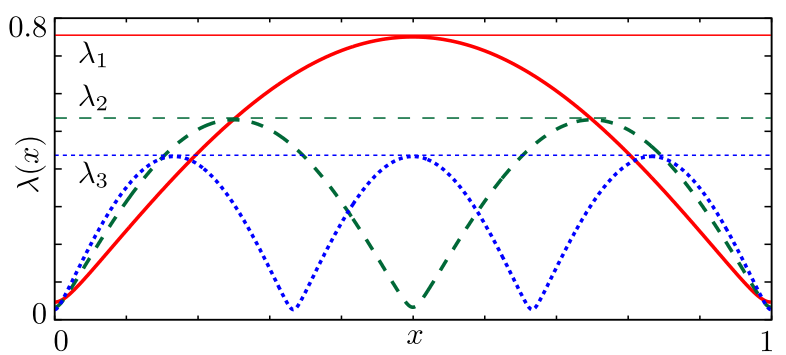

FIG. 6: (Color online) Local electron vibron coupling $\lambda(x)$ as a function of $x$ for $\delta=1, x_{v 0}=0$ and different vibron modes: red (solid) $p=1$, green (dashed) $p=2$, blue (dotted) $p=3$. The thin lines are the maxima of $\lambda(x)$. Other parameters: $\alpha=5, \lambda_{\mathrm{m}}=2, g=1$.

between the first three vibronic modes. The intensity of the electron-vibron coupling strength decreases with the increasing order of the vibronic mode. Denoting $\lambda_{p}$ the maximum of $\lambda(x)$ for the $p$ mode, we find $\lambda_{p}=\lambda_{1} / \sqrt{p}$. We now briefly comment on the value of the electron-

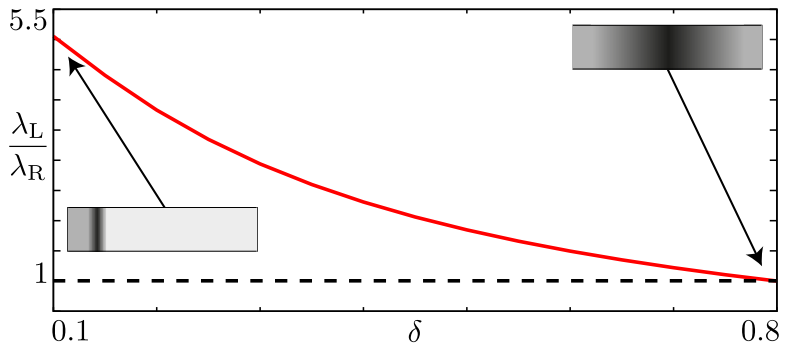

FIG. 7: (Color online) Ratio of the coupling strengths $\lambda_{\mathrm{L}} / \lambda_{\mathrm{R}}$ (see text) as a function of $\delta$ for a vibron with origin at $x_{v 0}=0.1 L$ and $p=1$. The shaded plots schematically depict the amplitude of the strain field of the vibronic mode $\hat{u}_{p}(x)$. Other parameters: $\alpha=5, \lambda_{\mathrm{m}}=2$ and $g=1$.

vibron coupling at the position of the tunneling barriers $\lambda_{\mathrm{L}} \equiv \lambda(0)$ and $\lambda_{\mathrm{R}} \equiv \lambda(L)$, which govern the lateral tun- neling rates. Figure 7 shows the ratio $\lambda_{\mathrm{L}} / \lambda_{\mathrm{R}}$ as a function of $\delta$ for a vibron with origin at $x_{v 0}=0.1 L$. At small values of $\delta$ the vibron is asymmetrically located near the left tunnel barrier. As a consequence, $\lambda_{\mathrm{L}}>\lambda_{\mathrm{R}}$. This mechanism is at the origin of the systematic suppression of conductance traces in a recent experiment $\stackrel{58}{58}$ For increasing $\delta$ the situation evolves towards a more symmetric setup and indeed for $\delta=0.8$, corresponding to a symmetric vibron with respect to the $\mathrm{CNT}$, one recovers $\lambda_{\mathrm{L}}=\lambda_{\mathrm{R}}$.

\section{B. Transport properties}

Figure 8 shows the density plot of the differential conductance $\mathcal{G}=\partial I / \partial V$ in the $\left(V, N_{\mathrm{g}}\right)$ plane for the situation depicted in Fig. 5(c), semiconducting case. The large white areas at small $V$ are the Coulomb

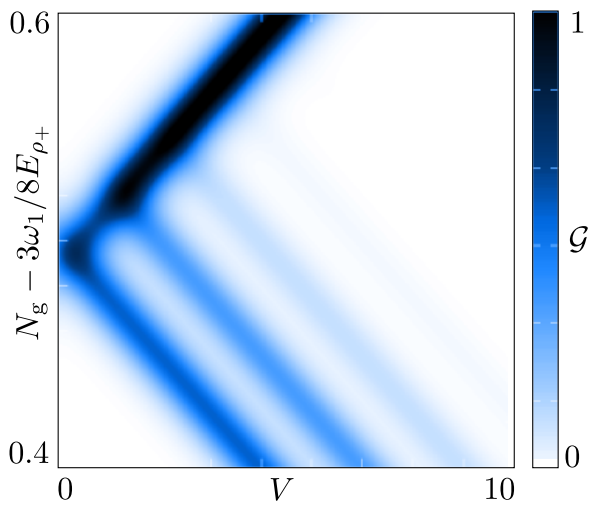

FIG. 8: (Color online) Semiconducting CNT. Differential conductance $\mathcal{G}$ (units $e^{2} \Gamma_{0}^{(\mathrm{T})} / \Omega_{0}$ ) as a function of $N_{\mathrm{g}}$ and $V$ (units $\Omega_{0} / e$ ) for a vibron originating at $x_{v 0}=0.15 L$ with $\delta=0.4$, $p=1$ and a tip at $x=0.35 \mathrm{~L}$. Other parameters: $\lambda_{\mathrm{m}}=2$, $\alpha=5, g=1, k_{\mathrm{B}} T=0.15 \Omega_{0}$ and $A=\Gamma_{0} / \Gamma_{0}^{(\mathrm{T})}=100$.

blockade regions where transport is interdicted and the CNT is occupied by $N_{0}$ or $N_{0}+1$ electrons. Within the transport region, delimited by the two most intense conductance traces, a series of equally spaced lines are clearly visible. They correspond to the excitation of the vibronic mode at energy $\Omega_{0}$. We consider different tunneling amplitudes through the tip and the lateral contacts, introducing the asymmetry $A=\Gamma_{0} / \Gamma_{0}^{(\mathrm{T})}$.

We will concentrate the discussion on the regime $k_{\mathrm{B}} T<$ $\Omega_{0}$, quoting for simplicity analytical expressions for $A \gg$ 1 only, realistic in an STM experiment. Exploiting the fact that $\lambda_{\mathrm{L}, \mathrm{R}} \ll 1$ we assume $B_{l}\left(x_{j}\right) \approx \delta_{l, 0}$. In the linear regime $(V \rightarrow 0)$ the conductance is then

$$
\mathcal{G}_{\operatorname{lin}} \approx \frac{2 \beta e^{2} \Gamma_{0}^{(\mathrm{T})} B_{0}(x)}{\cosh \left[\frac{\beta \Delta E}{2}-\frac{1}{2} \ln (4)\right] \cosh \left[\frac{\beta \Delta E}{2}\right]},
$$


with $\Delta E$ in Eq. (34). The logarithmic factor $\ln (4)$ stems from the fourfold degeneracy of the state with $N_{0}+1$ electrons. The amplitude of the linear conductance is modulated by the factor

$$
B_{0}(x)=e^{-\lambda^{2}(x)} .
$$

Therefore, the conductance is suppressed in the region where the electron-vibron coupling is large.

In the nonlinear regime $\left(V>k_{\mathrm{B}} T\right)$ one finds

$$
\mathcal{G}_{\text {nonlin }} \approx \frac{\beta e^{2} \Gamma_{0}}{2} \sum_{l \geq 0} \frac{B_{l}(x)}{\cosh \left[\beta \frac{\Delta E+l \Omega_{0}-e V / 2}{2}\right]} .
$$

Equation (39) represents a fan of equally-spaced conductance peak lines located at $E_{\rho_{+}}\left(1-2 N_{\mathrm{g}}\right)+3 \omega_{1} / 8 E_{\rho_{+}}+$ $l \Omega_{0}-\mathrm{eV} / 2=0$, thus with negative slope in the $\left(V, N_{\mathrm{g}}\right)$ plane, see Fig. 8 . They originate by the tunneling from the STM tip to the CNT, triggering vibronic excitations. We note that, because of the smallness of $\lambda_{\mathrm{L}, \mathrm{R}}$, the triggering process due to the tunneling on the contacts barriers is strongly suppressed (since $\left.B_{l}\left(x_{j}\right) \approx \delta_{l, 0}\right)$ with a corresponding absence of conductance lines with positive slope. Each of the above peaks is weighted by $B_{l}(x)$ which in turn conveys informations on $\lambda(x)$. This is particularly clear for the $l$-th $(l \geq 1)$ nonlinear conductance peak. Indeed, for $\lambda(x)<1$ (which is the case considered in our calculations, see Fig. 5 one finds that $B_{l}(x)$ is a monotonically increasing function of $\lambda(x)$ - see Eq. (35) - which implies an increase of the conductance for increasing coupling strength. This fact allows to directly map the spatial modulations of the nonlinear differential conductance into modulations of $\lambda(x)$.

To be more specific, let us consider the resonance case

$$
\Delta E=0 \Longleftrightarrow N_{\mathrm{g}}=\frac{1}{2}+\frac{3 \omega_{1}}{8 E_{\rho_{+}}}
$$

and study $\mathcal{G}$. As is clear by inspecting Figs. 9 (a-d), the differential conductance $\mathcal{G}$ exhibits position-dependent modulations in close agreement to the behavior of $\lambda(x)$. The most striking features show up indeed near the vibron position, where $\mathcal{G}$ is suppressed for $V \approx 0$, and is enhanced for $\mathrm{eV} \approx 2 l \Omega_{0}(l=1,2, \ldots)$, see Fig. 5 .

For the case of a metallic CNT, shown in Fig. 10 the spatial modulations of the conductance are less pronounced due to the decreased intensity of the electron-vibron coupling with respect to the semiconducting case.

The close resemblance of the spatial modulations of the non linear $\mathcal{G}$ with $\lambda(x)$ is supported studying the conductance at fixed bias shown in Fig. 11 for $e V=2 \Omega_{0}$ and $e V=4 \Omega_{0}$. Clearly $\mathcal{G}$ is enhanced where $\lambda(x)$ is large. This confirms that position-resolved conductance maps are source of valuable informations about the intensity of the strain field along the CNT and consequently on the location and size of the vibron mode.
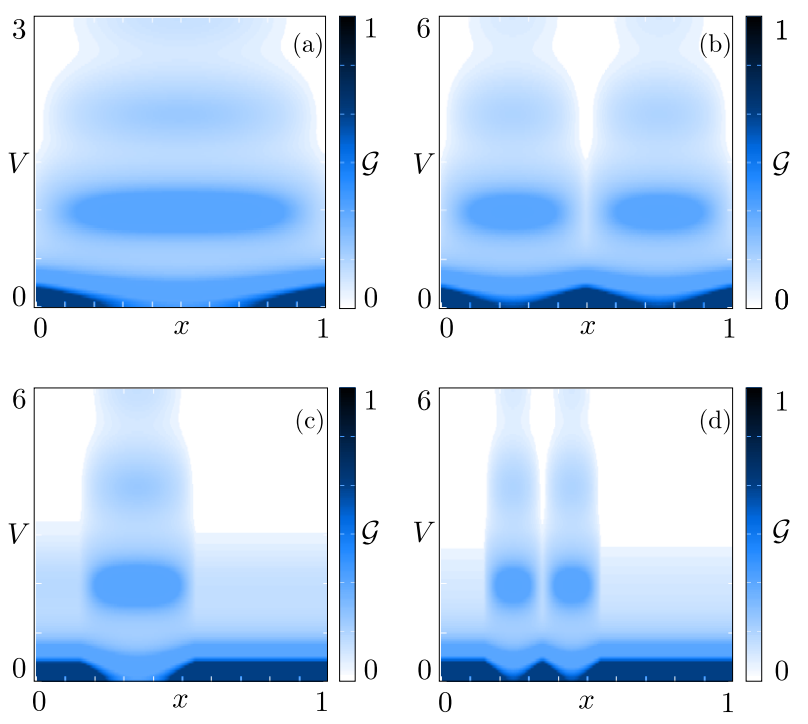

FIG. 9: (Color online) Semiconducting CNT: Plot of $\mathcal{G}$ (units $\left.e^{2} \Gamma_{0}^{(\mathrm{T})} / \Omega_{0}\right)$ as a function of the tip position $x$ and bias voltage $V$ (units $\Omega_{0} / e$ ) at resonance $N_{\mathrm{g}}=(1 / 2)+\left(3 \omega_{1} / 8 E_{\rho_{+}}\right)$and (a) $\delta=1, x_{v 0}=0$ and $p=1$; (b) same as in (a) but for $p=2$; (c) $\delta=0.4, x_{v 0}=0.15 L$ and $p=1$; (d) same as in (c) but for $p=2$. Other parameters: $\lambda_{\mathrm{m}}=2, \alpha=5, g=1$, $k_{\mathrm{B}} T=0.15 \Omega_{0}$ and $A=100$.
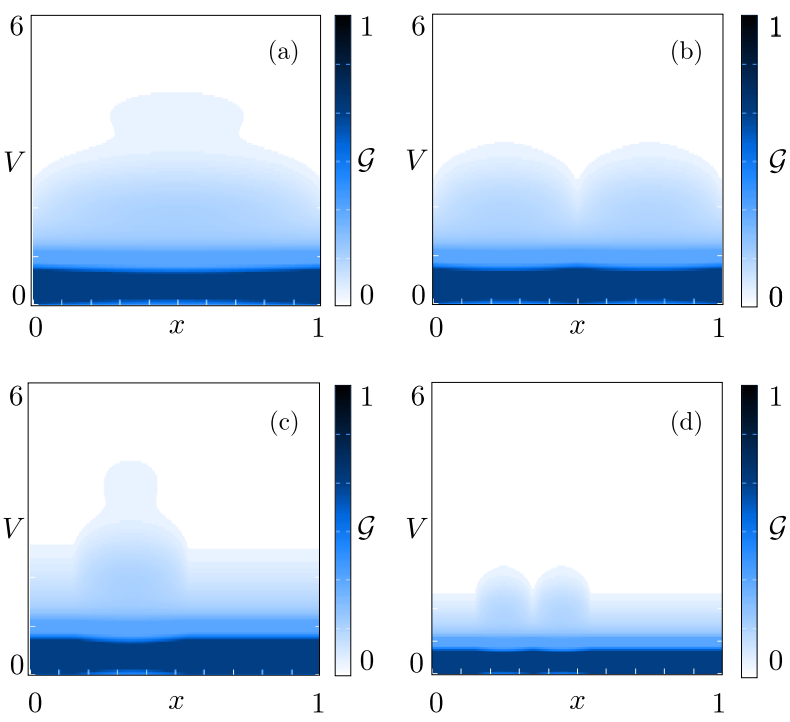

FIG. 10: (Color online) Metallic CNT: Plot of $\mathcal{G}$ (units $\left.e^{2} \Gamma_{0}^{(\mathrm{T})} / \Omega_{0}\right)$ as a function of the tip position $x$ and bias voltage $V$ (units $\Omega_{0} / e$ ) at resonance $N_{\mathrm{g}}=(1 / 2)+\left(3 \omega_{1} / 8 E_{\rho_{+}}\right)$and (a) $\delta=1, x_{v 0}=0$ and $p=1$; (b) same as in (a) but for $p=2$; (c) $\delta=0.4, x_{0}=0.15 L$ and $p=1$; (d) same as in (c) but for $p=2$. Other parameters: $\lambda_{\mathrm{m}}=2, \alpha=32, g=1$, $k_{\mathrm{B}} T=0.15 \Omega_{0}$ and $A=100$. 

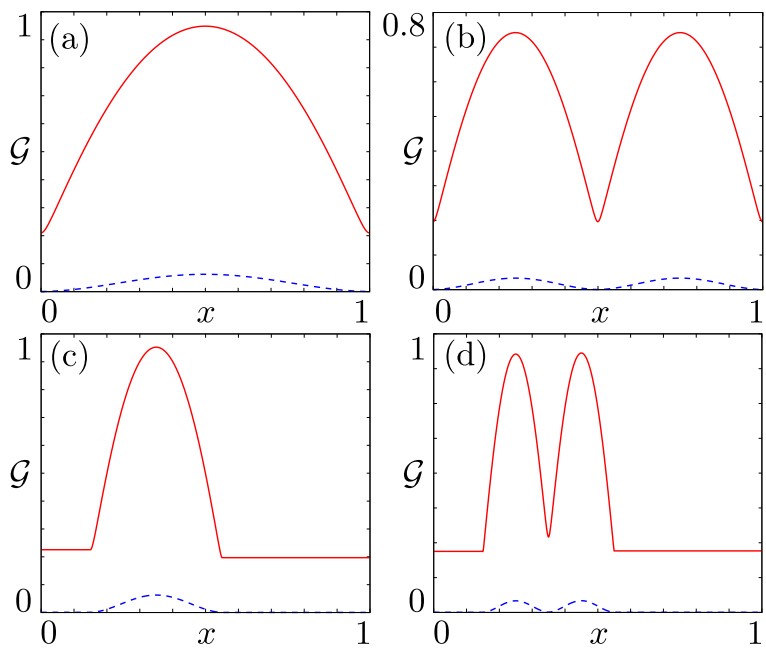

FIG. 11: (Color online) Differential conductance $\mathcal{G}$ (units $\left.e^{2} \Gamma_{0}^{(\mathrm{T})} / \Omega_{0}\right)$ as a function of the tip position $x$ for $N_{\mathrm{g}}=$ $(1 / 2)+\left(3 \omega_{1} / 8 E_{\rho_{+}}\right)$and $e V=2 \Omega_{0}$ (red solid line) or $e V=4 \Omega_{0}$ (blue dashed line) for a metallic CNT: (a) $\delta=1, x_{v 0}=0$ and $p=1$; (b) same as in (a) but for $p=2$; (c) $\delta=0.4$, $x_{v 0}=0.15 L$ and $p=1$; (d) same as in (c) but for $p=2$. Other parameters: $\lambda_{\mathrm{m}}=2, \alpha=32, g=1, k_{\mathrm{B}} T=0.15 \Omega_{0}$ and $A=100$.

\section{Interaction and asymmetry effects}

We close by briefly commenting about the role of electron-electron interactions and that of the barriers asymmetry.
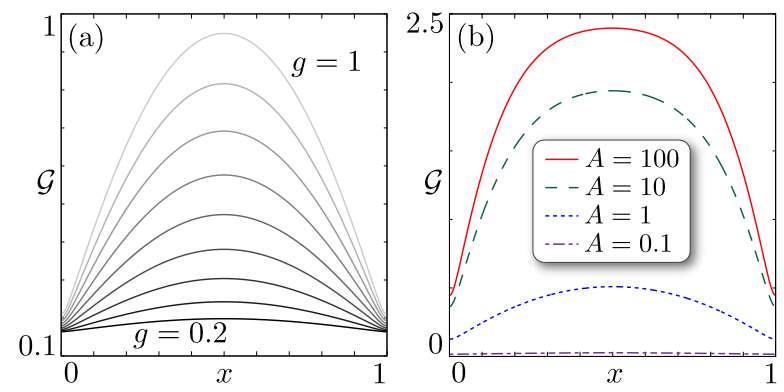

FIG. 12: (Color online) (a) Conductance $\mathcal{G}$ (units $e^{2} \Gamma_{0}^{(\mathrm{T})} / \Omega_{0}$ ) as a function of the tip position $x$ for decreasing values of $g$ from $g=1$ (noninteracting, lightest gray) to $g=0.2$ (strongly interacting, black); (b) Plot of $\mathcal{G}$ for different asymmetries (see key) and $g=1$. Other parameters: $\delta=1, x_{v 0}=0, p=1$, $\lambda_{\mathrm{m}}=2, \alpha=5, k_{\mathrm{B}} T=0.15 \Omega_{0}$ and $e V=2 \Omega_{0}$.
Figure 12(a) shows the position-resolved nonlinear conductance at $e V=2 \Omega_{0}$ for increasing Coulomb interaction strength (decreasing values of $g$ ). Clearly, conductance is suppressed in turns, signaling the suppression of $\lambda(x)$. This fact can be explained in terms of an increase of the velocity of the charged mode $v_{\rho_{+}}=v_{\mathrm{F}} / g$ which induces an effective parameter $\alpha$ higher than that of the noninteracting case.

Concerning the role of the asymmetry between the tip rate and the leads rate, Fig. 12(b) shows that the amplitude of the conductance modulations is decreased when making the tunnel barriers more symmetric with a collapse when reversing the asymmetry $A<1$ (i.e. making the contacts more opaque than the STM tip). This can be understood by observing that for $A \gg 1$ the current is dominated by the slowest barrier which is the STM one. This implies that the space-dependent tunneling rate can be efficiently probed in this regime.

\section{CONCLUSIONS}

In this paper we have shown how transport measurements, performed with a scanning tunnel microscope tip on a suspended carbon nanotube, can bring information about its vibrational stretching dynamics. This theory predicts a position-dependent coupling constant, which is larger in the region where the vibron is located.

The position-dependent coupling constant strongly affects the tunneling rate of electrons through the tip and has relevant consequences in the transport spectra. In particular, we showed that conductance maps in the linear and nonlinear regime, obtained sweeping the tip along the nanotube, are closely connected to the local coupling constant and allow to localize the position and size of the vibron. Effects can be more pronounced in semiconducting nanotubes due to the reduced Fermi velocity which matches more closely the speed of the vibrational mode. The role of electronic interactions and of the asymmetry between tip and metal contact tunnel barriers have also been addressed.

This work could inspire a new class of experiments which aim at studying the vibrational degrees of freedom of a nanotube by means of electrical measurements.

Acknowledgments. The authors acknowledge stimulating discussions with V. Cataudella, E. Mariani, A. Nocera, E. Paladino and C. Stampfer. Financial support by the EUFP7 via ITN-2008-234970 NANOCTM is also gratefully acknowledged.
1 S. Iijima, Nature (London) 354, 56 (1991).

2 J.-C. Charlier, X. Blase, and S. Roche, Rev. Mod. Phys. 79, 677 (2007).
${ }^{3}$ R. Saito, G. Dresselhaus, and M. S. Dresselhaus, 'Physical Properties of Carbon Nanotubes', Imperial College Press (1998). 
${ }^{4}$ D. H. Cobden and J. Nygård, Phys. Rev. Lett. 89, 046803 (2002).

5 M. Bockrath, D. H. Cobden, P. L. McEuen, N. G. Chopra, A. Zettl, A. Thess, and R. E. Smalley, Science 725, 1922 (1997).

${ }^{6}$ S. J. Tans, M. H. Devoret, H. Dai, A. Thess, R. E. Smalley, L. J. Geerligs, and C. Dekker, Nature 386, 474 (1997).

7 H. W. C. Postma, T. Teepen, Z. Yao, M. Grifoni, and C. Dekker, Science 293, 76 (2001).

8 A. K. Hüttel, G. A. Steele, B. Witkamp, M. Poot, L. P. Kouwenhoven, and H. S. J. van der Zant, Nano Lett. 9, 2547 (2009)

9 C. Stampfer, A. Jungen, R. Linderman, D. Obergfell, S. Roth, and C. Hierold, Nano Lett. 6, 1449 (2006).

10 M. Poot and H. S. J. van der Zant, to appear on Phys. Rep., arXiv:1106.2060v1.

11 B. J. Leroy, S. G. Lemay, J. Kong, and C. Dekker, Appl. Phys. Lett. 84, 4280 (2004).

12 B. J. LeRoy, S. G. Lemay, J. Kong, and C. Dekker, Nature 432, 371 (2004).

13 B. J. LeRoy, I. Heller, V. K. Pahilwani, C. Dekker, and S. G. Lemay, Nano Lett. 7, 2937 (2007).

14 V. Sazonova, Y. Yaish, H. Üstünel, D. Roundy, T. A. Arias, and P. L. McEuen, Nature 431, 284 (2004).

15 S. Sapmaz, P. Jarillo-Herrero, Y. M. Blanter, C. Dekker, and H. S. J. van der Zant, Phys. Rev. Lett. 96, 026801 (2006).

16 A. K. Hüttel, B. Witkamp, M. Leijnse, M. R. Wegewijs, and H. S. J. van der Zant, Phys. Rev. Lett. 102, 225501 (2009).

17 R. Leturcq, C. Stampfer, K. Inderbitzin, L. Durrer, C. Hierold, E. Mariani, M. G. Schultz, F. von Oppen, and K. Ensslin, Nat. Phys. 5, 327 (2009).

18 A. K. Hüttel, H. B. Meerwaldt, G. A. Steele, M. Poot, B. Witkamp, L. P. Kouwenhoven, and H. S. J. van der Zant, Phys. Status Solidi B 247, 2974 (2010).

19 G. A. Steele, A. K. Hüttel, B. Witkamp, M. Poot, H. B. Merrwaldt, L. P. Kouwenhoven, and H. S. J. van der Zant, Science 325, 1103 (2009).

${ }^{20}$ H. Lin, J. Lagoute, V. Repain, C. Chacon, Y. Girard, F. Ducastelle, H. Amara, A. Loiseau, P. Hermet, L. Henrard, and S. Rousset, Phys. Rev. B 81, 235412 (2010).

21 J. Lee, S. Eggert, H. Kim, S.-J. Kahng, H. Shinohara, and Y. Kuk, Phys. Rev. Lett. 93, 166403 (2004).

22 Y.-F. Chen, T. Dirks, G. Al-Zoubi, N. O. Birge, and N. Mason, Phys. Rev. Lett. 102, 036804 (2009).

23 W. Clauss, D. J. Bergeron, M. Freitag, C. L. Kane, E. J. Mele, and A. T. Johnson, Europhys. Lett. 47, 601 (1999).

24 M. Furuhashi and T. Komeda, Phys. Rev. Lett. 101, 185503 (2008).

25 L. C. Venema, J. W. G. Wilder, J. W. Janssen, S. J. Tans, H. L. J. T. Tuinstra, L. P. Kouwenhoven, and C. Dekker, Science 283, 52 (1999).

26 S. G. Lemay, J. W. Janssen, M. van den Hout, M. Mooij, M. J. Bronikowski, P. A. Willis, R. E. Smalley, L. P. Kouwenhoven, and C. Dekker, Nature (London) 412, 617 (2001).

27 M. Ouyang, J.-L. Huang, and C. M. Lieber, Phys. Rev. Lett. 88, 066804 (2002).

28 G. Buchs, D. Bercioux, P. Ruffieux, P. Gröning, H. Grabert, and O. Gröning, Phys. Rev. Lett. 102, 245505 (2009).

29 S. H. Abedinpour, M. Polini, G. Xianlong, and M. P. Tosi,
Phys. Rev. A 75, 015602 (2007).

30 J. Qian, B. I. Halperin, and E. J. Heller, Phys. Rev. B 81, 125323 (2010).

31 U. De Giovannini, F. Cavaliere, R. Cenni, M. Sassetti, and B. Kramer, Phys. Rev. B 77, 035325 (2008).

32 F. Cavaliere, U. De Giovannini, M. Sassetti, and B. Kramer, New J. Phys. 11, 123004 (2009).

33 T. Giamarchi, Quantum Physics in One Dimension, Oxford Science Publications (2004).

34 R. Egger and A. O. Gogolin, Phys. Rev. Lett. 79, 5082 (1997).

35 D. Bercioux, G. Buchs, H. Grabert, and O. Gröning, Phys. Rev. B 83, 165439 (2011).

36 S. Eggert, Phys. Rev. Lett. 84, 4413 (2000).

37 A. Crépieux, R. Guyon, P. Devillard, and T. Martin, Phys. Rev. B 67, 205408 (2003).

38 A. V. Lebedev, A. Crépieux, and T. Martin, Phys. Rev. B 71, 075416 (2005).

39 C. Bena, Phys. Rev. B 82, 035312 (2010).

40 H. Suzuura and T. Ando, Phys. Rev. B 65, 235412 (2002).

41 A. De Martino and R. Egger, Phys. Rev. B 67, 235418 (2003).

42 G. D. Mahan, Phys. Rev. B 68, 125409 (2003).

43 G. Pennington and N. Goldsman, Phys. Rev. B 68, 045426 (2003).

44 K. Flensberg, New J. Phys. 8, 5 (2006).

45 E. Mariani and F. von Oppen, Phys. Rev. B 80, 155411 (2009).

46 M. Verissimo-Alves, R. B. Capaz, B. Koiller, E. Artacho, and H. Chacham, Phys. Rev. Lett. 86, 3372 (2001).

47 W. Izumida and M. Grifoni, New J. Phys. 7, 244 (2005).

48 A. Zazunov, D. Feinberg, and T. Martin, Phys. Rev. B 73, 115405 (2006).

49 X. Y. Shen, B. Dong, X. L. Lei, and N. J. M. Horing, Phys. Rev. B 76, 115308 (2007).

50 J. Franck, Trans. Faraday Soc. 21, 536 (1926).

51 E. Condon, Phys. Rev. 28, 1182 (1926).

52 S. Braig and K. Flensberg, Phys. Rev. B 68, 205324 (2003).

53 J. Koch, F. von Oppen, and A. V. Andreev, Phys. Rev. B 74, 205438 (2006).

54 J. Koch and F. von Oppen, Phys. Rev. Lett. 94, 206804 (2005).

55 F. Haupt, F. Cavaliere, R. Fazio, and M. Sassetti, Phys. Rev. B 74, 205328 (2006).

56 M. Merlo, F. Haupt, F. Cavaliere, and M. Sassetti, New J. Phys. 10, 023008 (2008).

57 F. Cavaliere, G. Piovano, M. Sassetti, and E. Paladino, New J. Phys. 10, 115004 (2008)

58 F. Cavaliere, E. Mariani, R. Leturcq, C. Stampfer, and M. Sassetti, Phys. Rev. B 81, 201303(R) (2010).

59 H. Yoshioka and Y. Okamura, J. Phys. Soc. Jpn. 71, 2512 (2002).

60 M. Grifoni and L. Mayrhofer, Eur. Phys. J. B 56, 107 (2007).

61 T. Kleimann, F. Cavaliere, M. Sassetti, and B. Kramer, Phys. Rev. B 66, 165311 (2002).

62 F. Cavaliere, A. Braggio, J. T. Stockburger, M. Sassetti, and B. Kramer, Phys. Rev. Lett. 93, 036803 (2004).

${ }^{63}$ F. Cavaliere, A. Braggio, M. Sassetti, and B. Kramer, Phys. Rev. B 70, 125323 (2004).

64 R. A. Jishi, M. S. Dresselhaus, and G. Dresselhaus, Phys. Rev. B 48, 11385 (1993).

65 K. I. Bolotin, K. J. Sikes, J. Hone, H. L. Stormer, and P. Kim, Phys. Rev. Lett. 101, 096802 (2008). 
66 G. Piovano, F. Cavaliere, E. Paladino, and M. Sassetti, Phys. Rev. B 83, 245311 (2011).

67 P. Ullersma, Physica (Amsterdam) 32, 27 (1966).

68 A. Metelmann and T. Brandes, arXiv:1107.3762 (2011).
69 A. Donarini, A. Yar, and M. Grifoni, arXiv:1109.0723 (2011). 